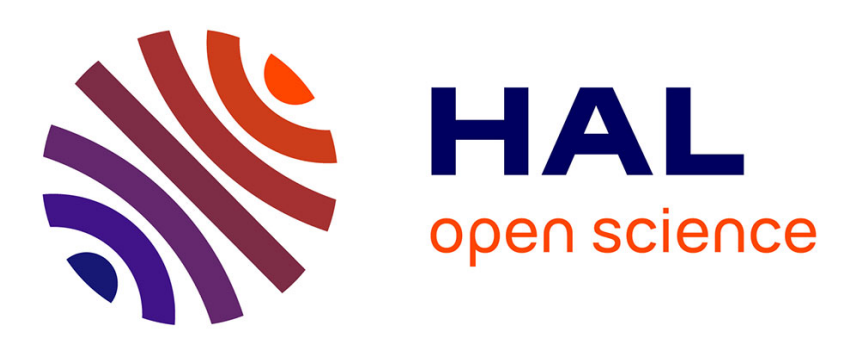

\title{
Quantum-Chemical Simulation of Solid-State NMR Spectra: The Example of a Molecular Tweezer Host-Guest Complex
}

\author{
Jan Zienau, Joerg Kussmann, Christian Ochsenfeld
}

\section{- To cite this version:}

Jan Zienau, Joerg Kussmann, Christian Ochsenfeld. Quantum-Chemical Simulation of Solid-State NMR Spectra: The Example of a Molecular Tweezer Host-Guest Complex. Molecular Physics, 2010, 108 (03-04), pp.333-342. 10.1080/00268970903476647 . hal-00580673

\section{HAL Id: hal-00580673 \\ https://hal.science/hal-00580673}

Submitted on 29 Mar 2011

HAL is a multi-disciplinary open access archive for the deposit and dissemination of scientific research documents, whether they are published or not. The documents may come from teaching and research institutions in France or abroad, or from public or private research centers.
L'archive ouverte pluridisciplinaire HAL, est destinée au dépôt et à la diffusion de documents scientifiques de niveau recherche, publiés ou non, émanant des établissements d'enseignement et de recherche français ou étrangers, des laboratoires publics ou privés. 


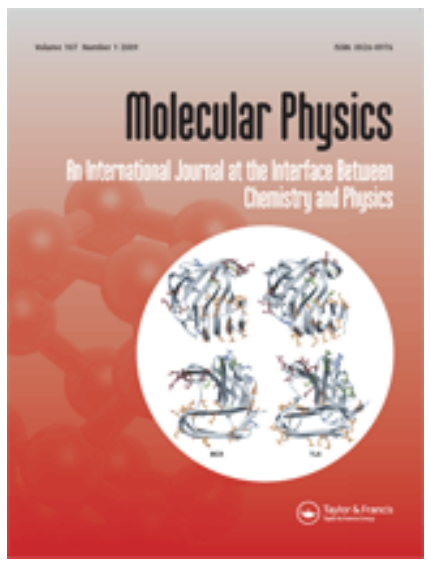

\section{Quantum-Chemical Simulation of Solid-State NMR Spectra: The Example of a Molecular Tweezer Host-Guest Complex}

\begin{tabular}{|c|c|}
\hline Journal: & Molecular Physics \\
\hline Manuscript ID: & TMPH-2009-0258.R1 \\
\hline Manuscript Type: & Special Issue Paper - In honour of Prof Werner 60th birthday \\
\hline $\begin{array}{r}\text { Date Submitted by the } \\
\text { Author: }\end{array}$ & 04-Nov-2009 \\
\hline Complete List of Authors: & $\begin{array}{l}\text { Zienau, Jan; University of Tuebingen } \\
\text { Kussmann, Joerg; University of Tuebingen } \\
\text { Ochsenfeld, Christian; University of Tuebingen, Chemistry }\end{array}$ \\
\hline Keywords: & $\begin{array}{l}\text { quantum chemistry, linear scaling, NMR, molecular recognition, } \\
\text { molecular tweezer }\end{array}$ \\
\hline \multicolumn{2}{|c|}{$\begin{array}{l}\text { Note: The following files were submitted by the author for peer review, but cannot be converted } \\
\text { to PDF. You must view these files (e.g. movies) online. }\end{array}$} \\
\hline ochsenfeld_TMPH-2009-0258 & 3.R1.tar.gz \\
\hline
\end{tabular}

\section{(s) ScholarONE" \\ Manuscript Central}




\title{
Quantum-Chemical Simulation of Solid-State NMR
}

\section{Spectra: The Example of a Molecular Tweezer Host-Guest Complex}

\author{
Jan Zienau, Jörg Kussmann, \\ and Christian Ochsenfeld* \\ Institut für Physikalische und Theoretische Chemie, \\ Auf der Morgenstelle 8, Universität Tübingen, D-72076 Tübingen, Germany
}

\begin{abstract}
A systematic quantum-chemical study of the convergence of proton NMR shieldings with the size of solid-state fragments is presented for a host-guest system. The largest system computed at Hartree-Fock and density-functional theory levels comprises a full first shell of complexes surrounding a central unit within an X-ray based structure and a total of 1196 atoms and 13260 basis functions. While the influence of methodological aspects can be considered to be converged within the error bars of experiment and theory, the deviation of one of the protons provides evidence for the possibility of a dynamic rotation process of the guest within the host complex.
\end{abstract}

* Author to whom correspondence should be sent: Fax: +49-7071-29-5490, e-mail: christian.ochsenfeld@uni-tuebingen.de 


\section{Introduction}

Nuclear magnetic resonance (NMR) spectroscopy is one of the most important experimental methods for gaining insights not only into the atomistic structure of molecular systems, but also into some aspects of their dynamical behavior [1,2]. Despite this tremendous success, the lack of a direct relationship between the measured NMR signals and the structure of a molecule raises the need for reliable assignments of the experimental spectra. Here, quantum-chemical calculations can provide important insights (see e.g. Refs.[3-5]).

While quantum chemistry has evolved over the last decades to become a very valuable tool for computing molecular properties including NMR chemical shifts [5], the applicability to larger molecular systems has been hampered by the strong, at least cubic, increase of the computational cost with molecular size. To overcome these limitations, methods were introduced whose cost scales only linearly with molecular size (see e.g. Ref. [6] and references therein), so that systems in the range of 1000 atoms are computable even on today's oneprocessor computers.

For the ab-initio calculation of NMR chemical shieldings this scaling wall was overcome only recently [7-9], so that important new possibilities for studying molecular NMR spectra for large systems with 1000 and more atoms open up. This has not only interesting implications for the study of NMR spectra of large molecules, but also for describing the 'environment' of a molecule in solution or the solid state.

In our present work, we focus on studying the influence of the solid-state environment on the NMR chemical shifts of a host-guest complex. By means of our linear-scaling gaugeincluding atomic-orbital Hartree-Fock (GIAO-HF) and GIAO-density functional theory (GIAODFT) methods $[7,8]$, we are for the first time able to systematically increase the fragment size of the solid-state structure and to account for all the neighboring complexes of a chosen host-guest complex. Here, we focus on a molecular tweezer system synthesized in the Klärner group [10] which binds a dicyanobenzene guest molecule and can be employed as useful model system for molecular recognition processes. Since an X-ray structure is available for this system [10], it is ideally suited for studying the possibilities of assigning NMR spectra by means of quantum-chemical approaches. In order to account for the full neighboring sphere of a host-guest complex, we consider as largest fragment a system with 1196 atoms and 13260 basis functions without molecular symmetry.

\section{Computational Details}

For all calculations a development version of the Q-CHEM program package [11] was used. Structures of the solid-state fragments of the dicyanobenzene-tweezer complex were obtained by optimizing the monomer at the $\mathrm{HF} / 6-31 \mathrm{G}^{*}$ level with constraints from the X-ray structure [10] and subsequently projecting the monomer units onto the experimentally maintained positions in the solid-state [12]. SCF calculations were carried out employing linear-scaling 
1

methods for calculating the Fock matrix: continuous fast multipole method (CFMM) [13] and linear exchange (LinK) method [14,15]. For the DFT calculations we employed the BP86(VWN) functional [16-18] with the SG-1 grid [19] for the numerical integrations [20]. NMR calculations were performed using the $6-31 \mathrm{G}^{* *}[21,22]$ basis with our linear-scaling method [7,8] at the GIAO-HF [23-27] and GIAO-DFT [28-34] levels, respectively. Relative NMR shifts are given, unless noted otherwise, with respect to those of a TMS molecule calculated at the same level of theory. For experimental details concerning the X-ray structure and the magic-angle spinning (MAS) experiment see Ref. [10]. It should be mentioned that the crystallographic structure data were obtained at a temperature of $298 \mathrm{~K}$ whereas the NMR spectrum was recorded at $321 \mathrm{~K}$. 


\section{Simulation of Solid-State NMR Spectra}

The reliable simulation of solid-state NMR spectra by quantum-chemical methods poses three major challenges: (1) Structure determination of building blocks (monomers) and their arrangement in the solid phase must be followed by (2) convergence studies of the NMR data with respect to fragment size, where it is also necessary (3) to estimate error bars caused by the chosen quantum-chemical method and basis set (see as well discussion in Refs. [12,35]).

While we were able to gain useful information by quantum-chemical simulation of NMR spectra for a variety of similar systems $[6,10,12,35-42]$, we focus in our present work on the study of a dicyanobenzene (DCNB) tweezer host-guest complex (see Fig. 1) and compare the experimentally observed MAS-NMR proton shieldings to quantum-chemically calculated shieldings for different fragment sizes of the solid-state structure. The DCNB system has already been subject to earlier studies by us $[10,12,35]$, where we were constrained to rather small fragments such as a trimer at most. Due to the availability of our linear-scaling code for the calculation of NMR shifts we are now able to perform full DFT and HF calculations for fragment sizes of up to a tridecamer, i.e., a DCNB tweezer monomer with the full first sphere of neighboring complexes, without having to resort to an incremental scheme [12] or other additional approximations. In this way, we study the convergence of calculated proton NMR chemical shieldings at DFT and HF levels, where we expect that the major effects of neighboring groups are sufficiently accounted for by the tridecamer. This corresponds to the second challenge mentioned above, whereas the first and third challenges for the present system have been discussed earlier $[12,35]$ and will be briefly re-examined below.

\subsection{Structures of the Investigated Fragments}

The structures employed for the NMR calculations of the solid-state fragments (first challenge discussed above) are based on an X-ray structure [12]. Since proton positions were not determined in the experimental structure, the structure of the monomer unit was optimized at the $\mathrm{HF} / 6-31 \mathrm{G}^{*}$ level, while several constraints were imposed as described in Ref. [12] in order to account for the lack of dispersion forces at the HF level and for missing influences occurring in the solid-state environment. The (partially) optimized monomer structure was afterwards projected onto the corresponding positions in the solid-state structure.

Just as a side note, it is clear that a theoretically more satisfying alternative to this pragmatic approach would be a full geometry optimization of a unit cell of the solid-phase crystal employing a sufficiently accurate method that accounts for dispersion-type effects. In the past, this was prevented due to the prohibitive scaling properties of conventional post-HF methods and the failure of traditional DFT functionals to handle dispersive interactions. Here, recent developments in particular in the field of low-order scaling Møller-Plesset perturbation theory for the calculation of energies and gradients [43-48], but also the design of dispersioncorrected DFT functionals (see e.g. Refs. [49,50] and Refs. [51,52] for applications) seem 
to offer promising perspectives. However, as these methodologies are mostly still topics of active developments, the structures used in this study were all obtained using the pragmatic approach described above, which also ensures comparability to earlier studies $[10,12,35]$.

In our present work, we focus on the second aspect discussed above for simulating a solidstate NMR spectrum, namely to study the convergence with respect to the fragment size employed for modeling the solid phase. It is worthwhile to note, that besides this 'cluster approach' an alternative approach for simulating solid-state NMR spectra would be the use of periodic boundary conditions for describing the solid phase [53-56]. While we have currently not explored this approach so far, there has been some success described in the literature for smaller systems in other context (see e.g. Ref. [57]).

\subsection{Monomer Host-Guest Complex and Methodological Accura- cies}

The host-guest complex of the naphthalene-spaced tweezer host and the dicyanobenzene guest, which represents the monomer unit, is schematically depicted in Fig. 1. In terms of the accuracy of the chosen method and basis set (third challenge mentioned above) we have found in earlier quantum-chemical studies that for a given molecular structure of the isolated dicyanobenzene its proton shieldings computed at the GIAO-HF/6-31G** and the GIAO-BP86(VWN)/6-31G** levels agree within 0.0 and $0.3 \mathrm{ppm}$, respectively, with our most reliable GIAO-MP2/QZ2P data [35]. Upon binding the dicyanobenzene molecule within the tweezer host, the ring-currents of the aromatic units of the tweezer influence the proton shieldings of the guest by up to 5-6 ppm [35]. Using a model consisting of the DCNB and one benzene molecule of the central sidewall of the tweezer host, we compared our most reliable GIAO-MP2/SVP data for this system with the GIAO-HF/6-31G** and GIAOBP86(VWN)/6-31G** results and found deviations of 0.0 and $0.5 \mathrm{ppm}$ for proton $\mathrm{H}_{b}$ facing the benzene unit, respectively [35]. The underestimation of ring-current influences using small basis sets such as 6-31G** at the DFT level is found as well for other functionals and reduces only for larger basis sets (and hybrid functionals which include more HF exchange; see discussion in Ref. [35]). Despite these disadvantages compared to Hartree-Fock, the GIAO-BP86(VWN)/6-31G** method has the important benefit of being computationally significantly less demanding than the GIAO-HF/6-31G** method, since no iterative solution of the CPSCF equations is required. We therefore consider it reasonable to further estimate the usefulness of the BP86(VWN) method in the context of the present study, so that we computed the NMR shifts mostly at both GIAO-HF/6-31G** and GIAO-BP86(VWN)/6$31 \mathrm{G}^{* *}$ levels of theory (see Table 1 and Fig. 1 for numbering). As a side note, it would also be interesting to explore, in the present context, the performance of other DFT functionals developed specifically for the calculation of NMR chemical shifts, such as the ones of Keal and Tozer $[58,59]$. While we plan to carry out such investigations in the future, they are, however, not in focus of the present work. 


\subsection{Convergence of NMR Shifts with Respect to Fragment Size}

Prior to the present work, we have performed studies on monomer, dimer, and trimer units of the host-guest complex using the HF method with a variety of basis sets $[10,12]$. At that time no linear-scaling NMR code [7] had been developed, so that we were constrained to using small basis sets for the dimer and trimer fragments. We therefore employed an incremental scheme based on our ab-initio data, by adding the influences of neighboring units onto the monomer values (see Ref. [12] for a detailed discussion). This approach did not only allow to estimate environmental influences, but as well was expected to profit from an error cancellation for the rather small basis sets employed. The accuracy of this incremental approach was recently discussed for a fragment of five tweezer units with a tetracyano-pquinodimethane (TCNQ) guest $[7,35]$.

In Table 1 we list the computed ${ }^{1} \mathrm{H}-\mathrm{NMR}$ chemical shifts (GIAO-HF/6-31G** and GIAOBP86(VWN)/6-31G**) for monomer, dimer, trimer, heptamer, and tridecamer units in comparison to the experimental solid state MAS-NMR data. Here, we analyze the shieldings calculated at the HF level first, before discussing the BP86(VWN) results. If we consider initially the relative chemical shifts of the monomer, the comparison to the experiment shows already a good agreement for the guest protons (deviations of 0.1 and $0.2 \mathrm{ppm}$ ), while it should be kept in mind that the error bars in both experiment and theory are expected to be in the order of $0.2-0.4 \mathrm{ppm}$ [12] for the present systems. The only protons which are clearly outside the error bars of experiment and theory are those at the tip of the tweezer $\left(\mathrm{H}_{2 / 3 / 14 / 15}\right.$, see Fig. 1) with a deviation of $1.7 \mathrm{ppm}$. As discussed earlier by us $[10,12,60]$ the reason is obvious if a larger fragment of the solid state is considered: The corresponding protons are located directly above the naphthalene system of the neighboring host-guest complex so that strong ring-current influences are expected. Therefore, the major part can already be accounted for by employing a dimer fragment (Table 1): The chemical shifts of the corresponding protons in unit A of the dimer (which corresponds to just the units A and B of the trimer shown in Fig. 4) change by 1.4-1.5 ppm as compared to the monomer values and then agree within $0.2-0.3 \mathrm{ppm}$ with the experimental value. These values do not change much $(0.2$ ppm at most) in proceeding from the dimer to the trimer system (Tab. 1 and Fig. 4), which shows that unit $\mathrm{C}$ in this fragment has only a minor influence on the corresponding shieldings of A.

As we have pointed out in Ref. [12] it is clear that in order to truly account for all influences in the solid state, we need to include at least the first shell of host-guest complexes around a specific complex, which leads to the tridecamer (13 host-guest complexes, 1196 atoms) depicted in Fig. 6. The NMR chemical shifts computed at the GIAO-HF level are listed in Tab. 1. All protons in the tridecamer except $\mathrm{H}_{a}$ deviate only by $0.2 \mathrm{ppm}$ at most when comparing them to the trimer fragment, so that these protons can be considered to be converged with respect to the fragment size already for the trimer. For guest proton $\mathrm{H}_{a}$, however, the data show a large effect onto the chemical shieldings by $0.9 \mathrm{ppm}$ in the 
tridecamer as compared to the monomer, while the corresponding influences for proton $\mathrm{H}_{a}$ within the dimer and trimer models studied so far are only small in the order of $0.3 \mathrm{ppm}$.

The reason for this large effect onto the guest proton $\mathrm{H}_{a}$ is obvious if one considers the large tridecamer fragment: It is due to the relatively close position of two neighboring guest systems (within the guest plane). Although the dimer/trimer models which we have studied in Ref. [12] allow to reliably account for the major ring current effects influencing the neighboring complexes, this guest-guest interaction has not been accounted for so far. As compared to the intramolecular ring current effects within the host-guest complex influencing the guest protons $\mathrm{H}_{a}$ and $\mathrm{H}_{b}$ by 2.4 and 5.7 ppm, respectively (GIAO-HF/6-31G**; compare Tables 1 and 3 of Ref. [35]), the present effect of $0.9 \mathrm{ppm}$ is smaller but nevertheless very important for the description.

In order to study this influence on the guest proton $\mathrm{H}_{a}$ in more detail, we performed calculations focusing only onto the guest molecules of the three central 'face-on' complexes within the tridecamer. The interaction of three guest molecules without the tweezers (structures and structural positions of the guests are kept unchanged) leads to a change of the $\mathrm{H}_{a}$ chemical shift by $1.4 \mathrm{ppm}$ at the GIAO-HF/6-31G** level compared to the isolated guest, while $\mathrm{H}_{b}$ is influenced by $0.1 \mathrm{ppm}$ at most. The same values hold if the calculations are performed at the GIAO-BP86(VWN)/6-31G** level. These values remain virtually unchanged (0.1 ppm) if just two guest molecules are considered instead of three. If the tweezer hosts are also included in the above mentioned 'face-on' trimer, which leads to the 'face-on' trimer host-guest complex fragment depicted in Fig. 3, the influence on proton $\mathrm{H}_{a}$ of the guest of the central complex is very similar (1.5 ppm as compared to the monomer host-guest complex), whereas the influence on $\mathrm{H}_{b}$ is slightly larger $(0.4 \mathrm{ppm})$ as when considering the guest molecules only.

The larger influence onto the guest proton $\mathrm{H}_{a}$ of $1.4 / 1.5 \mathrm{ppm}$ in considering the 'face-on' structures (without/with the tweezer hosts) instead of $0.9 \mathrm{ppm}$ for the entire tridecamer has to be due to compensation effects of the tweezer complexes neglected in the 'face-on' systems. These effects are also illustrated by comparing the monomer data to the corresponding data for complex A of the heptamer (see Fig. 5 and Tab. 1). Here, no guest 'face-on' effects are included, so that the difference in the chemical shift for $\mathrm{H}_{a}$ when comparing monomer and heptamer should be solely due to the compensation effects mentioned above. As can be seen from Tab. 1, the chemical shift of the proton $\mathrm{H}_{a}$ changes from the monomer value of 5.5 ppm to $5.0 \mathrm{ppm}$ in the heptamer, yielding a shift change of $0.5 \mathrm{ppm}$ which is similar to the difference of the influences discussed above (1.4/1.5 ppm vs. $0.9 \mathrm{ppm})$. The consideration of the heptamer therefore explains the partial compensation of the guest 'face-on' influence in the tridecamer.

While we have so far focused on NMR shieldings computed at the GIAO-HF/6-31G** level, it is worthwhile to discuss the corresponding GIAO-BP86(VWN)/6-31G** shifts as listed in Tab. 1. Here, the difference in the relative shieldings compared to HF is $0.3 \mathrm{ppm}$ at most for all given protons except for $\mathrm{H}_{b}$, where the deviation is significantly larger with 0.5 to $0.6 \mathrm{ppm}$. As already reported earlier [35], the BP86(VWN) functional shows for smaller basis 
sets such as 6-31G** an inferior description of the (non-local) ring-current effects influencing this proton as compared to HF. Therefore the GIAO-HF/6-31G** method is expected to be more reliable than the GIAO-BP86(VWN)/6-31G** method for the present systems.

\subsection{Tridecamer Fragment}

So far we have discussed the convergence behavior of the computed NMR data with respect to increasing fragment size, so that it remains to compare the chemical shifts of our best model for the solid-state, the tridecamer, to those of the experiment. The GIAO-HF/6$31 \mathrm{G}^{* *}$ and the GIAO-BP86(VWN)/6-31G** methods yield an agreement with the MAS-NMR data within $0.5 \mathrm{ppm}$ and $0.6 \mathrm{ppm}$, respectively, for all protons except $\mathrm{H}_{a}$, where the deviation is in the order of $0.9 \mathrm{ppm}$ for both methods and therefore outside the estimated experimental and theoretical error bars (see above).

As a first attempt to explain this disagreement, one may assume problems associated with the chosen TMS reference, that can occur for both the quantum-chemical calculation and the solid-state MAS-NMR experiment: The quantum-chemical difficulty is related to the often strongly different electronic structure of TMS as compared to the one of the molecular system of interest. This is well known to be a problem, in particular if ${ }^{13} \mathrm{C}-\mathrm{NMR}$ chemical shifts are considered (see e.g. Ref. [12]). For the latter the use of intermediate reference systems has proven to be quite successful [12]. Also in the MAS experiment several difficulties may arise, since TMS cannot be used as internal standard, but instead often adamantane or other compounds [61]. This has been discussed, e.g., in Ref. [61] as possible source of difficulties. As a consequence, it often proves useful to entirely avoid the external TMS standard, but instead to compare just the pattern of the NMR spectrum in employing an internal standard for comparing experiment and theory.

If one selects, e.g., $\mathrm{H}_{b}$ as internal reference, we obtain the values shown in Tab. 2. Although the corresponding GIAO-BP86(VWN)/6-31G** results are in good agreement with the experimental solid-state NMR data with deviations of $0.6 \mathrm{ppm}$ at most $\left(\mathrm{H}_{\text {Bridgehead }}\right)$, this good agreement is in large parts fortuitious, since the GIAO-BP86(VWN)/6-31G** approach is known to underestimate ring-current influences (see Ref. [35]). For the more reliable GIAO$\mathrm{HF} / 6-31 \mathrm{G}^{* *}$ method such a nice agreement as observed at the BP86 level is not found: At the $\mathrm{HF}$ level, the tridecamer data computed with respect to $\mathrm{H}_{b}$ as internal reference shows a strong maximum difference to the experiment $(0.9 \mathrm{ppm}$, similar to the data obtained without internal standard). This means that the maximum deviation between theory and experiment in the order of $0.9 \mathrm{ppm}$ persists, independently whether the internal or the external TMS reference is employed.

In order to analyze this discrepancy between theory and experiment in more detail, it is important to reconsider the accuracies expected at this stage of the investigation: (1) the GIAO-HF /6-31G** method was shown earlier to provide a good description of proton chemical shifts in the present systems [35], (2) choosing an internal standard does not alter the observed 
disagreement between experimental and GIAO-HF/6-31G** data, and (3) choosing an even larger fragment, i.e. incorporating the second shell, is expected to have only minor influences on the shifts of the central host-guest complex as compared to those of the 13-mer. Therefore it seems that methodological reasons for the observed discrepancy of $0.9 \mathrm{ppm}$ between theory and experiment can be excluded.

A possible explanation for the discrepancy of the $\mathrm{H}_{a}$ shieldings could be the static description of the guest position in the X-ray structure employed as the basis for the quantumchemical NMR calculations. As observed earlier by means of MAS-NMR measurements at higher temperatures (coalescence of $\mathrm{H}_{a}$ and $\mathrm{H}_{b}$ signals at $410 \mathrm{~K}$ ) [10], a rotation of the DCNB guest within the tweezer cavity can occur. In principle, two rotation modes are possible: Either a rotation around the $\mathrm{C}_{2}$ axis perpendicular to the benzene plane of DCNB denoted as 'in-plane-rotation' in the following, or a rotation around the axis through the CN units of DCNB denoted as 'CN-rotation' (see also Fig. 2 for an illustration of the rotations). While a full (60 degrees) in-plane-rotation can be excluded within the solid-state due to space constraints (collision of neighboring CN groups during the rotation process; see discussion in Ref. [12]), a small oscillation is always possible. Even though the MAS-NMR data discussed in our present work were recorded at a lower temperature $(321 \mathrm{~K})$ than the coalescence temperature mentioned above, a slight rotation by a few degrees around the crystallographically determined positions at $298 \mathrm{~K}$ is conceivable for both modes.

In order to evaluate the influence of such rotations on the calculated NMR shieldings, we chose as a model system the 'face-on' trimer host-guest complex fragment depicted in Fig. 3, which represents a cutout of the central unit of the three 'face-on' complexes in the tridecamer. We subsequently estimated the changes in chemical shifts when rotating the guest molecules starting from their crystallographically determined positions around both axes by angles $\vartheta$ and $\varphi$, respectively (for definition of angles see Fig. 2). Here, all three guest molecules were rotated in the same manner, i.e., 'synchronously'. The in-plane-rotation was performed so that the N-N distances of neighboring guest molecules were enlarged during rotation (i.e., clockwise in the projection of Fig. 6 (b)); the CN-rotation is quasi symmetric concerning the rotation direction. The resulting shift changes for the central tweezer of the 'face-on' trimer fragment were computed at the GIAO-HF/6-31G** level for two/three different angles (see Tab. 3).

For all the considered rotation angles an upfield-shifting influence on the NMR shielding of $\mathrm{H}_{a}$ is observed. Table 3 shows that both a CN-rotation of $22^{\circ}$ or an in-plane-rotation of only $5^{\circ}$ causes upfield changes of about $0.4 \mathrm{ppm}$ for $\mathrm{H}_{a}$. The chemical shifts of the other given protons remain virtually unaffected by the $5^{\circ}$ in-plane-rotation, whereas they are mostly less influenced than $\mathrm{H}_{a}$ for the other listed cases, and are, if at all, shifted slightly downfield (0.3 ppm at maximum) when the average values are considered.

We note in passing that analogous calculations at the GIAO-BP86(VWN)/6-31G** level for the example of an in-plane-rotation angle of $5^{\circ}$ yield the same behavior. We also tested the influence of the $5^{\circ}$ in-plane-rotation on all other protons except $\mathrm{H}_{a}$ of the central tweezer 
(not only the ones given in Tab. 3) and found only negligible effects (0.1 ppm at most).

As can be seen from Table 3 and already discussed above, rotations around both axes lead to an upfield-shifting influence on the shielding of $\mathrm{H}_{a}$, whereas the other protons are less influenced and show in part slight down-field shifts. Although at higher temperatures with larger rotation angles the CN-rotation process should be favored over the in-plane-rotation due to space constraints within the solid state (see Ref. [12] where ' $180^{\circ}$ rotation' refers to the full 180 degrees CN-rotation and '60 rotation' refers to the full 60 degrees in-plane rotation process), this statement is not necessarily true for small values of rotation angles which we expect at lower temperatures. However, solely on the basis of our computed NMR values a distinction of both processes is not possible.

In order to illustrate the influence of the rotation upon the total shieldings of the 13-mer, we added the shift changes upon the guest in-plane-rotation of $5^{\circ}\left(\Delta_{r o t}\left(5^{\circ}\right)\right)$ computed for the 'face-on' trimer to the computed shieldings of the tridecamer. This seems justified since we do not expect the additional complexes within the 13-mer, as compared to the 'face-on' trimer, to exert a significant influence on these shift changes. The resulting incremented tridecamer chemical shifts are displayed in the penultimate column of Table 1. These 'rotationally corrected' values show a much better overall agreement between theory and experiment within $0.5 \mathrm{ppm}$ at the HF and $0.6 \mathrm{ppm}$ at the DFT level for all protons, which is within the experimental and theoretical error bars. The good agreement suggests that such rotational processes might be expected to be of importance for describing the MAS-NMR spectrum recorded at $321 \mathrm{~K}$, while the two rotation processes cannot be distinguished within our present study.

\section{Conclusion}

In this work, we have performed quantum-chemical NMR calculations to simulate a solidstate MAS-NMR spectrum of a host-guest system by systematically studying the convergence of proton chemical shifts upon increasing the size of solid-state fragments. As model system a dicyanobenzene tweezer host-guest complex is considered for which an X-ray structure is available. The largest fragment computed at the GIAO-HF/6-31G** and GIAO-BP86(VWN)/6$31 \mathrm{G}^{* *}$ levels comprises a full shell of neighboring complexes around a central host-guest unit and a total of 13 tweezer complexes with 1196 atoms and 13260 basis functions.

Although the shieldings of the central host-guest unit are expected to be largely converged, it is only one guest proton $\left(\mathrm{H}_{a}\right)$ which shows a larger deviation of about $0.9 \mathrm{ppm}$ as compared to the experiment. The agreement of theory and experiment for the shieldings of the remaining protons is within $0.5 / 0.6 \mathrm{ppm}$ and in this way well within the error bars of both experiment and theory estimated to be in the order of $0.2-0.4 \mathrm{ppm}$, respectively. Since the computed data is expected to be widely independent of methodological deficiencies, our detailed study suggests that the discrepancy for the $\mathrm{H}_{a}$ guest proton can be attributed to the static nature of the X-ray structure employed for the quantum-chemical calculation of the NMR shieldings. 
1

Taking into account some degree of guest dynamical processes, some of which were already observed earlier in different context for higher temperatures [10], we observe that a slight rotatory distortion can explain the deviation between theory and experiment. In this way, the NMR results obtained upon taking into account a rotation process lead to a satisfactory accordance of theory and experiment for all considered protons. Therefore, we conclude that such guest dynamics may indeed play a role already at the temperature where the MAS-NMR measurements were made.

\section{Acknowledgments}

The authors thank Dr. F. Koziol for preparing the projected fragment structures during his graduate studies in our group. Furthermore, we thank Prof. Dr. F.-G. Klärner (Universität Duisburg-Essen, Germany), Prof. Dr. H.W. Spiess (MPIP Mainz, Germany), and Dr. S.P. Brown (University of Warwick, England) for fruitful collaborations on the DCNB complex. C.O. acknowledges financial support by an Emmy Noether research grant of the DFG ("Deutsche Forschungsgemeinschaft"). J.Z. thanks the 'Landesgraduiertenförderung' (LGFG) for a graduate fellowship. 


\section{References}

[1] R.R. Ernst; Angew. Chemie Int. Ed. 31, 805 (1992).

[2] K. Wüthrich; Angew. Chemie Int. Ed. 42, 3340 (2003).

[3] T. Helgaker, M. Jaszunski and K. Ruud; Chem. Rev. 99, 293 (1999).

[4] M. Kaupp, M. Bühl and V.G. Malkin (Eds.); Calculation of NMR and EPR Parameters Wiley-VCH, Weinheim (2004).

[5] J. Gauss; in J. Grotendorst; Modern Methods and Algorithms of Quantum Chemistry NIC Series, pp. 541-592, John von Neumann Institute for Computing, Jülich, Second Edition (2000).

[6] C. Ochsenfeld, J. Kussmann and D.S. Lambrecht; in K.B. Lipkowitz und T.R. Cundari; Reviews in Computational Chemistry Vol. 23, pp. 1-82, VCH Publishers, New York, (2007).

[7] C. Ochsenfeld, J. Kussmann and F. Koziol; Angew. Chemie Int. Ed. 43, 4485 (2004).

[8] J. Kussmann and C. Ochsenfeld; J. Chem. Phys. 127, 054103 (2007).

[9] M. Beer and C. Ochsenfeld; J. Chem. Phys. 128, 221102 (2008).

[10] S.P. Brown, T. Schaller, U.P. Seelbach, F. Koziol, C. Ochsenfeld, F.-G. Klärner and H.W. Spiess; Angew. Chemie Int. Ed. 40, 717 (2001).

[11] Development version of the Q-Chem program package (http://www.q-chem.com); 2009.

[12] C. Ochsenfeld, F. Koziol, S.P. Brown, T. Schaller, U.P. Seelbach and F.-G. Klärner; Solid State Nucl. Magn. Reson. 22, 128 (2002).

[13] C.A. White, B.G. Johnson, P.M.W. Gill and M. Head-Gordon; Chem. Phys. Lett. 230, 8 (1994).

[14] C. Ochsenfeld, C.A. White and M. Head-Gordon; J. Chem. Phys. 109, 1663 (1998).

[15] C. Ochsenfeld; Chem. Phys. Lett. 327, 216 (2000).

[16] A.D. Becke; Phys. Rev. A 38, 3098 (1988).

[17] J.P. Perdew; Phys. Rev. B 33, 8822 (1986).

[18] S.H. Vosko, L. Wilk and M. Nusair; Can. J. Phys. 58, 1200 (1980).

[19] P.M.W. Gill, B.G. Johnson and J.A. Pople; Chem. Phys. Lett. 209, 506 (1993). 
[20] B. G. Johnson, C. A. White, Q. Zhang, B. Chen, R. L. Graham, P. M. W. Gill and M. Head-Gordon; in J. M. Seminario; Recent Developments in Density Functional Theory Vol. 4, p. 441, Elsevier Science, Amsterdam (1996).

[21] W.J. Hehre, R. Ditchfield and J.A. Pople; J. Chem. Phys. 56, 2257 (1972).

[22] P.C. Hariharan and J.A. Pople; Theor. Chim. Acta 28, 213 (1973).

[23] H.F. Hameka; Mol. Phys 1, 203 (1958).

[24] M. Häser, R. Ahlrichs, H.P. Baron, P. Weis and H. Horn; Theor. Chim. Acta 83, 455 (1992).

[25] F. London; J. Phys. Radium 8, 397 (1937).

[26] R. Ditchfield; Mol. Phys 27, 789 (1974).

[27] K. Wolinski, J.F. Hinton and P. Pulay; J. Am. Chem. Soc. 112, 8251 (1990).

[28] T. Helgaker, P.J. Wilson, R.D. Amos and N.C. Handy; J. Chem. Phys. 113, 2983 (2000).

[29] V.G. Malkin, O.L. Malkina and D.R. Salahub; Chem. Phys. Lett. 204, 80 (1993).

[30] V.G. Malkin, O.L. Malkina, M.E. Casida and D.R. Salahub; J. Am. Chem. Soc. 116, 5898 (1994).

[31] G. Schreckenbach and T. Ziegler; J. Phys. Chem. 99, 606 (1995).

[32] G. Rauhut, S. Puyear, K. Wolinski and P. Pulay; J. Phys. Chem. 100, 6310 (1996).

[33] J.R. Cheeseman, G.W. Trucks, T.A. Keith and M.J. Frisch; J. Chem. Phys. 104, 5497 (1996).

[34] A.M. Lee, N.C. Handy and S.M. Colwell; J. Chem. Phys. 103, 10095 (1995).

[35] J. Zienau, J. Kussmann, F. Koziol and C. Ochsenfeld; Phys. Chem. Chem. Phys 9, 4552 (2007).

[36] C. Ochsenfeld; Phys. Chem. Chem. Phys 2, 2153 (2000).

[37] C. Ochsenfeld, S.P. Brown, I. Schnell, J. Gauss and H.W. Spiess; J. Am. Chem. Soc. 123, 2597 (2001).

[38] W. Pisula, Z. Tomovic, M.D. Watson, K. Müllen, J. Kussmann, C. Ochsenfeld, T. Metzroth and J. Gauss; J. Phys. Chem. 111, 7481 (2007).

[39] M. Fokkens, C. Jasper, T. Schrader, F. Koziol, C. Ochsenfeld, J. Polkowska, M. Lobert, B. Kahlert and F.-G. Klärner; Chem. Eur. J. 11, 477 (2005). 
[40] F.-G. Klärner, B. Kahlert, A. Nellesen, J. Zienau, C. Ochsenfeld and T. Schrader; J. Am. Chem. Soc. 128, 4831 (2006).

[41] P. Rzepecki, K. Hochdörffer, T. Schaller, J. Zienau, K. Harms, C. Ochsenfeld, X. Xie and T. Schrader; J. Am. Chem. Soc. 130, 586 (2008).

[42] J. Polkowska, F. Bastkowski, T. Schrader, F.-G. Klärner, J. Zienau, F. Koziol and C. Ochsenfeld; J. Phys. Org. Chem. 22, 779 (2009).

[43] M. Schütz, G. Hetzer and H.-J. Werner; J. Chem. Phys. 111, 5691 (1999).

[44] C. Pisani, L. Maschio, S. Casassa, M. Halo, M. Schütz and D. Usvyat; J. Comput. Chem. 29, 2113 (2008).

[45] D.S. Lambrecht, B. Doser and C. Ochsenfeld; J. Chem. Phys. 123, 184102 (2005).

[46] B. Doser, D.S. Lambrecht, J. Kussmann and C. Ochsenfeld; J. Chem. Phys. 130, 064107 (2009).

[47] S. Schweizer, B. Doser and C. Ochsenfeld; J. Chem. Phys. 128, 154101 (2008).

[48] J. Zienau, L. Clin, B. Doser and C. Ochsenfeld; J. Chem. Phys. 130, 204112 (2009).

[49] Y. Zhao, N.E. Schultz and D.G. Truhlar; J. Chem. Theory Comput. 2, 364 (2006).

[50] S. Grimme; J. Comput. Chem. 25, 1463 (2004).

[51] B.M. Wong; J. Comput. Chem. 30, 51 (2009).

[52] M. Parac, M. Etinski, M. Peric and S. Grimme; J. Chem. Theory Comput. 1, 1110 (2005).

[53] F. Mauri, B.G. Pfrommer and S.G. Louie; Phys. Rev. Lett. 77, 5300 (1996).

[54] C.J. Pickard and F. Mauri; Phys. Rev. B 63, 245101 (2001).

[55] D. Sebastiani and M. Parrinello; J. Phys. Chem. A 105, 1951 (2001).

[56] V. Weber, M. Iannuzzi, S. Giani, J. Hutter, R. Declerck and M. Waroquier; J. Chem. Phys. 131, 014106 (2009).

[57] J. Schmidt, A. Hoffmann, H.W. Spiess and D. Sebastiani; J. Phys. Chem. B 110, 23204 (2006).

[58] T.W. Keal and D.J. Tozer; J. Chem. Phys. 119, 3015 (2003).

[59] T.W. Keal and D.J. Tozer; J. Chem. Phys. 121, 5654 (2004).

[60] T. Schaller, U.P. Büchle, F.-G. Klärner, D. Bläser, S.P. Brown, H.W. Spiess, F. Koziol, J. Kussmann and C. Ochsenfeld; J. Am. Chem. Soc. 129, 1293 (2007). 
[61] C.R. Morcombe and K.W. Zilm; J. Mag. Res. 162, 479 (2003). 


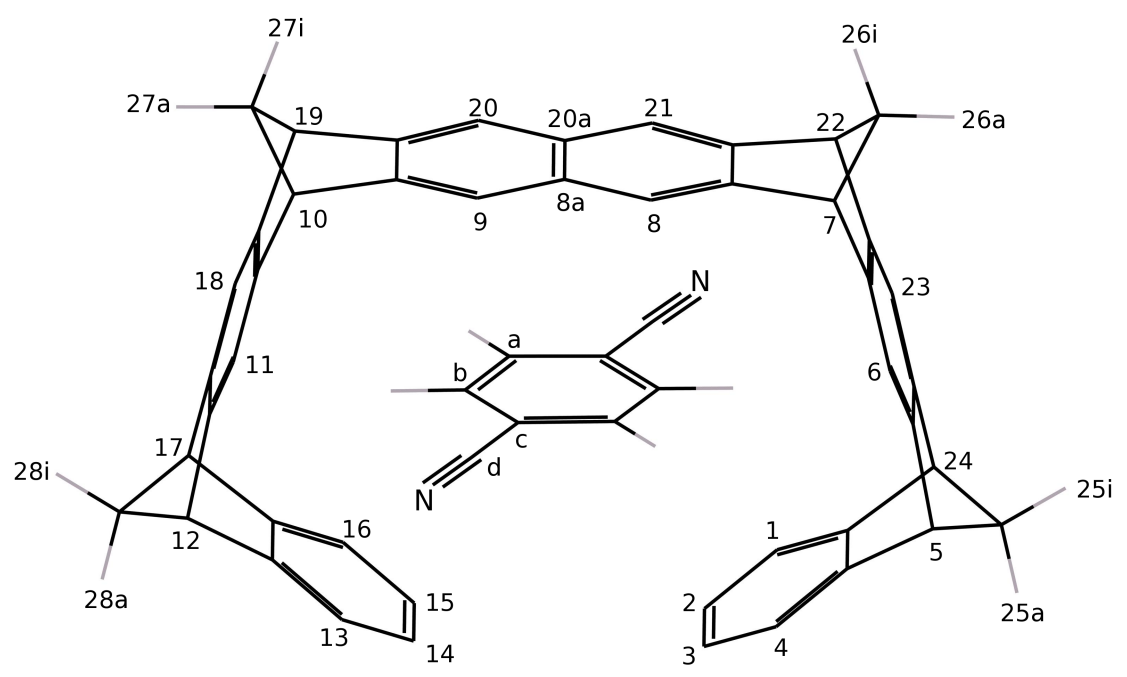

Figure 1: Schematic structure and numbering of the dicyanobenzene-tweezer complex. The label $\mathrm{H}_{\text {Bridgehead }}$ as used in tables and text refers to protons No. 5, 7, 10, 12, 17, 19, 22, and 24 . 

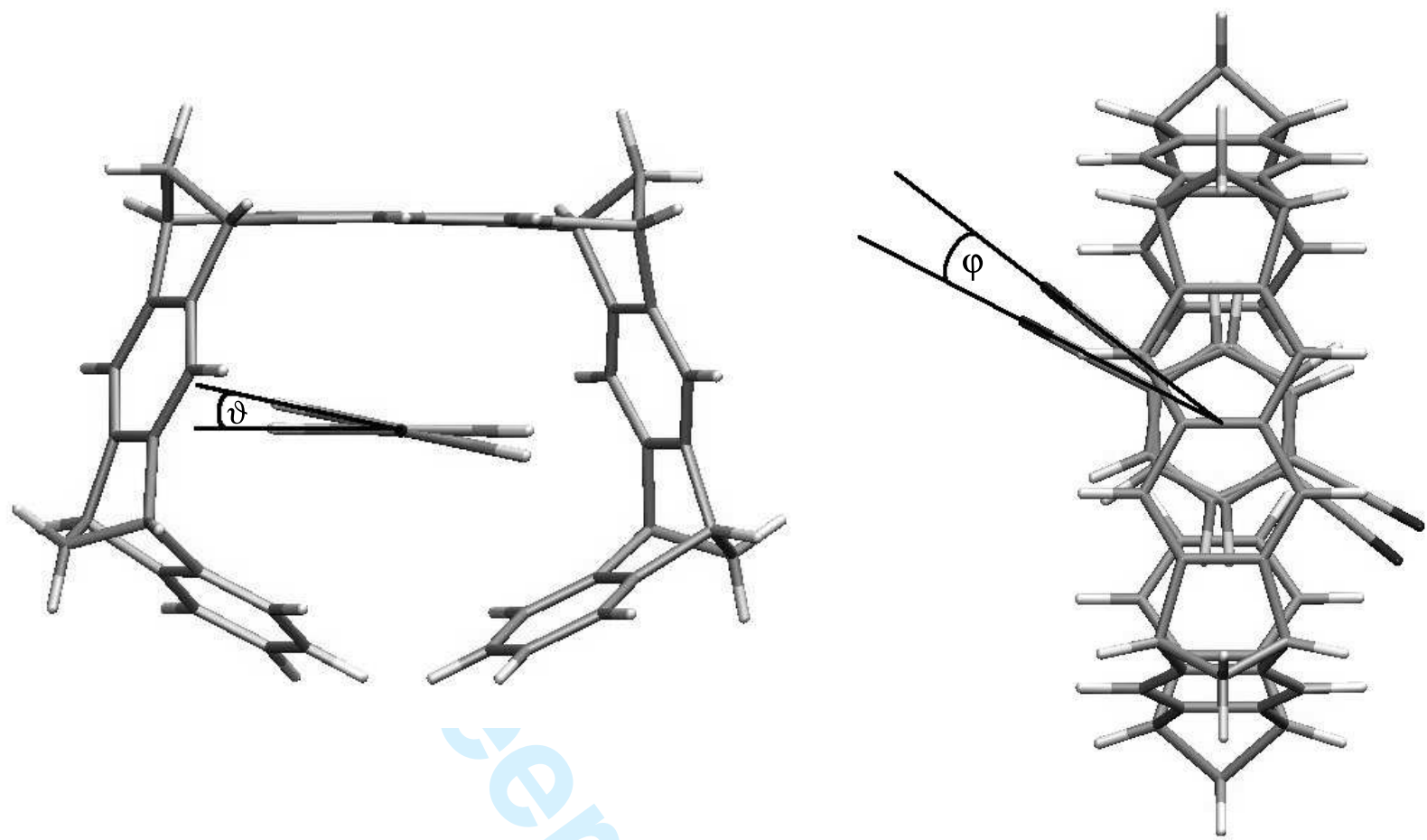

Figure 2: Definition of angles $\vartheta$ for the CN-rotation (left, view along C-N axis of DCNB guest) and $\varphi$ for the in-plane rotation (right, top view of tweezer) of the guest molecule, respectively.

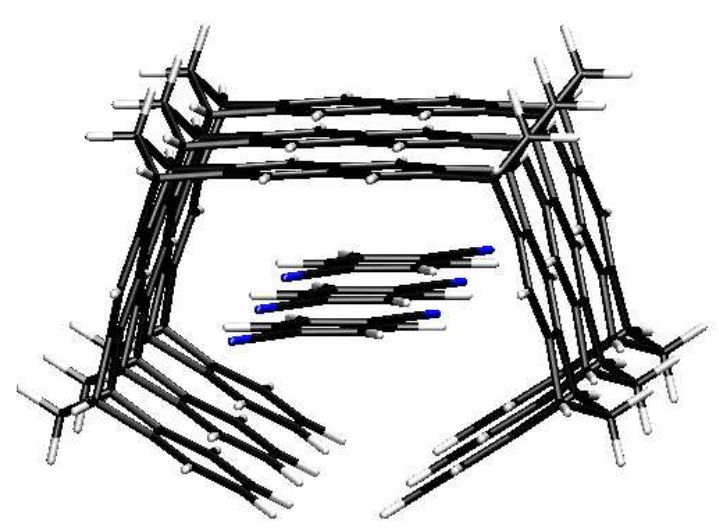

Figure 3: 'Face-on' trimer fragment. 


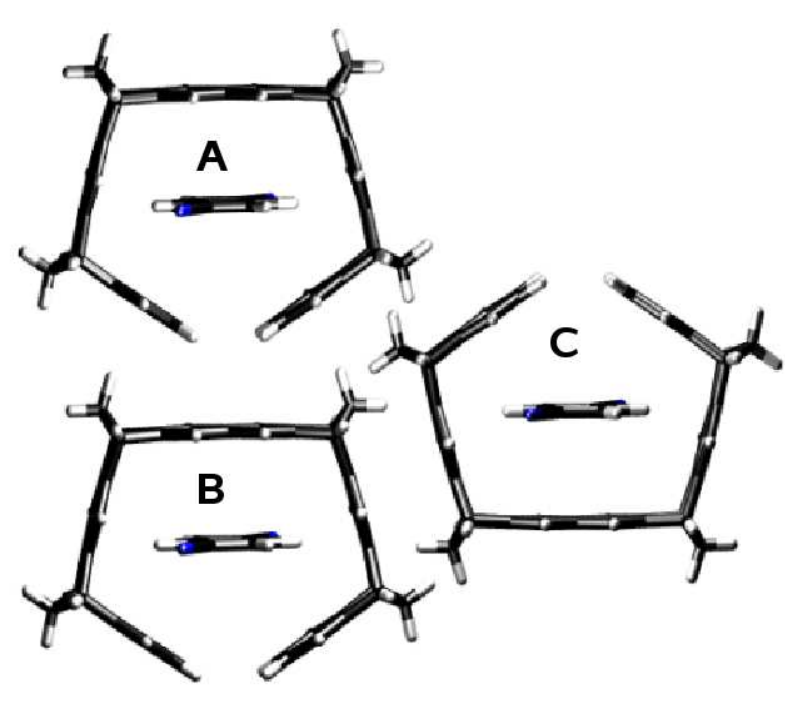

a)

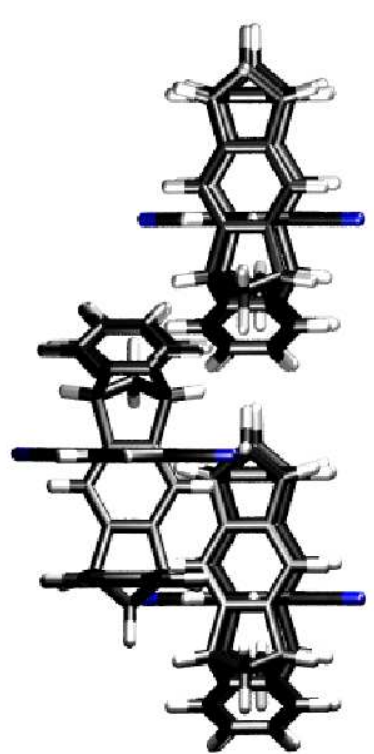

b)

Figure 4: Trimer fragment: a) Side view with labelling of the host-guest units. b) Side view rotated by 90 degrees.

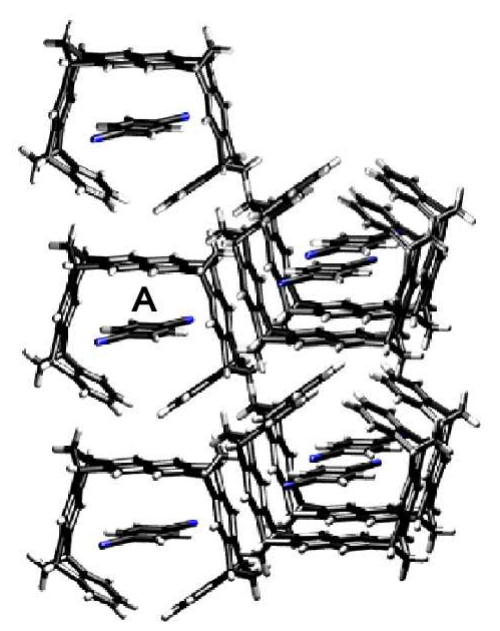

Figure 5: Heptamer fragment with unit A for which the shieldings are analyzed. 


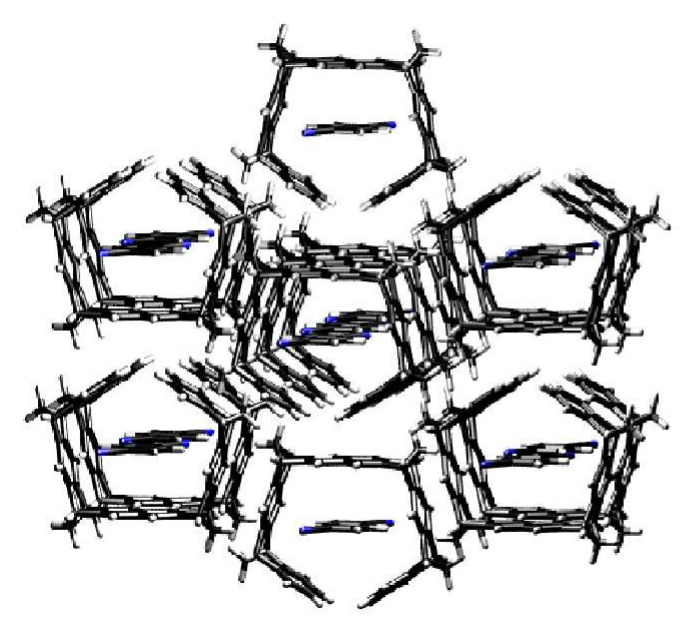

a)

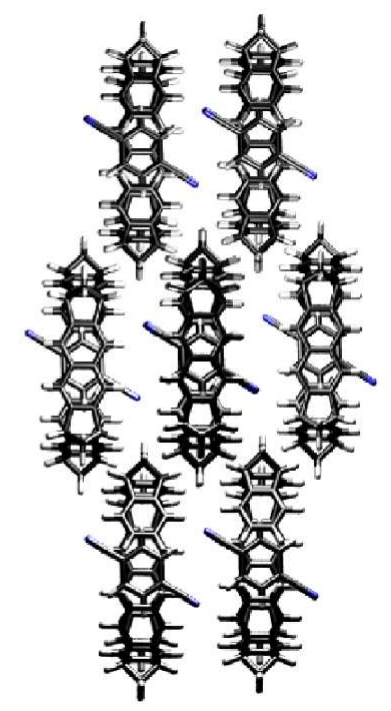

b)

Figure 6: Tridecamer fragment: a) Side view. b) Top view. 
Table 1: Relative isotropic NMR chemical shifts of selected protons for various fragment sizes up to a 13-mer (with 1196 atoms) with respect to TMS as obtained at the GIAO-HF/6-31G** and GIAO-BP86(VWN)/6-31G** levels. Structures of the trimer, heptamer, and tridecamer are shown in Fig. 4, Fig. 5, and Fig. 6. For the tridecamer all presented shifts are those calculated for the central tweezer complex. Here, $\Delta_{r o t}\left(5^{\circ}\right)$ means the increment of the chemical shift as obtained due to the in-plane-rotation of the guest molecule by $\varphi=5$ degrees in the 'face-on' trimer fragment (see Fig. 3 and Table 3).

\begin{tabular}{ll|c|c|c|c|c|c|c}
\hline \hline Method & & Monomer & Dimer & Trimer & Heptamer & \multicolumn{2}{|c|}{$\begin{array}{c}\text { Tridecamer } \\
+\Delta_{\text {rot }}\left(5^{\circ}\right)\end{array}$} & Exp. \\
\hline \hline HF & Proton & & $\mathrm{A}$ & $\mathrm{A}$ & $\mathrm{A}$ & & 6.0 & 5.6 \\
& $\mathrm{H}_{a}$ & 5.5 & 5.2 & 5.2 & 5.0 & 6.4 & $1.9-2.0$ & 2.0 \\
& $\mathrm{H}_{b}$ & 2.2 & 1.9 & 1.9 & $1.6-1.7$ & $1.9-2.0$ & $1.9-1.9$ \\
& $\mathrm{H}_{2 / 3 / 14 / 15}$ & 6.6 & $5.1-5.2$ & $5.1-5.3$ & $5.1-5.2$ & $5.3-5.4$ & $5.3-5.4$ & 4.9 \\
& $\mathrm{H}_{\text {Bridgehead }}$ & $3.9-4.1$ & $3.8-4.0$ & $3.6-4.1$ & $3.0-3.9$ & $3.5-4.3$ & $3.5-4.3$ & 3.8 \\
\hline BP86(VWN) & $\mathrm{H}_{a}$ & 5.5 & 5.3 & $5.2-5.3$ & 5.0 & 6.5 & 6.1 & 5.6 \\
& $\mathrm{H}_{b}$ & 2.8 & 2.5 & 2.5 & 2.2 & 2.5 & 2.5 & 2.0 \\
& $\mathrm{H}_{2 / 3 / 14 / 15}$ & $6.5-6.6$ & $5.4-5.5$ & $5.3-5.5$ & 5.3 & 5.5 & 5.5 & 4.9 \\
& $\mathrm{H}_{\text {Bridgehead }}$ & $4.0-4.2$ & $3.9-4.1$ & $3.8-4.1$ & $3.2-4.0$ & $3.7-4.3$ & $3.7-4.3$ & 3.8 \\
\hline \hline
\end{tabular}


Table 2: Relative isotropic NMR chemical shifts of selected protons for various fragment sizes up to a 13-mer (with 1196 atoms) with respect to proton $\mathrm{H}_{b}$ as obtained at the GIAO-HF/6-31G** and GIAO-BP86(VWN)/6-31G** levels. Structures of the trimer, heptamer, and tridecamer are shown in Fig. 4, Fig. 5, and Fig. 6. For the tridecamer all presented shifts are those calculated for the central tweezer complex.

\begin{tabular}{|c|c|c|c|c|c|c|c|}
\hline Method & Proton & Monomer & $\begin{array}{c}\text { Dimer } \\
\text { A }\end{array}$ & $\begin{array}{c}\text { Trimer } \\
\text { A }\end{array}$ & $\begin{array}{c}\text { Heptamer } \\
\text { A }\end{array}$ & Tridecamer & Exp. \\
\hline \multirow[t]{4}{*}{$\mathrm{HF}$} & $\mathrm{H}_{a}$ & 3.3 & 3.3 & 3.3 & 3.4 & 4.5 & 3.6 \\
\hline & $\mathrm{H}_{b}$ & 0.0 & 0.0 & 0.0 & $0.0-0.1$ & $0.0-0.1$ & 0.0 \\
\hline & $\mathrm{H}_{2 / 3 / 14 / 15}$ & 4.4 & $3.2-3.3$ & $3.2-3.4$ & $3.5-3.6$ & $3.4-3.5$ & 2.9 \\
\hline & $\mathrm{H}_{\text {Bridgehead }}$ & $1.7-1.9$ & $1.9-2.1$ & $1.7-2.2$ & $1.4-2.3$ & $1.6-2.4$ & 1.8 \\
\hline \multirow[t]{4}{*}{ BP86(VWN) } & $\mathrm{H}_{a}$ & 2.7 & 2.8 & $2.7-2.8$ & 2.8 & 4.0 & 3.6 \\
\hline & $\mathrm{H}_{b}$ & 0.0 & 0.0 & 0.0 & 0.0 & 0.0 & 0.0 \\
\hline & $\mathrm{H}_{2 / 3 / 14 / 15}$ & $3.7-3.8$ & $2.9-3.0$ & $2.8-3.0$ & 3.1 & 3.0 & 2.9 \\
\hline & $\mathrm{H}_{\text {Bridgehead }}$ & $1.2-1.4$ & $1.4-1.6$ & $1.3-1.6$ & $1.0-1.8$ & $1.2-1.8$ & 1.8 \\
\hline
\end{tabular}


Table 3: Relative isotropic NMR chemical shifts $(\delta)$ of selected protons for the central tweezer of the 'face-on' trimer fragment (see Fig. 3) with respect to TMS and corresponding changes $\Delta \delta$ of NMR chemical shifts upon rotation of the guest molecule around the CN axis (angle $\vartheta$ ) and the perpendicular $\mathrm{C}_{2}$ axis (angle $\varphi$ ) (see Fig. 2). All shifts were obtained at the GIAO-HF /6-31G** level.

\begin{tabular}{|c|c|c|c|c|c|c|}
\hline \multirow[b]{2}{*}{ Proton } & \multirow[t]{2}{*}{ " $\delta$} & \multicolumn{3}{|c|}{ 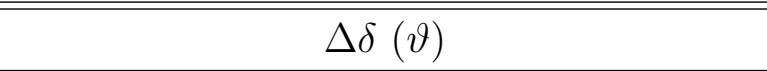 } & \multicolumn{2}{|c|}{ 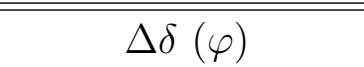 } \\
\hline & & $5^{\circ}$ & $11^{\circ}$ & $22^{\circ}$ & $5^{\circ}$ & $11^{\circ}$ \\
\hline $\mathrm{H}_{a}$ & 7.0 & $(-0.1)-(0.0)$ & $(-0.2)$ & $(-0.4)-(-0.5)$ & $(-0.4)$ & $(-0.6)$ \\
\hline $\mathrm{H}_{b}$ & 2.6 & $(-0.1)-(0.2)$ & $(-0.2)-(0.4)$ & $(0.1)-(0.3)$ & $(0.0)$ & $(0.3)$ \\
\hline $\mathrm{H}_{2 / 3 / 14 / 15}$ & 6.8 & $(-0.1)-(0.1)$ & $(-0.1)-(0.2)$ & $(-0.1)-(0.4)$ & $(0.0)$ & $(0.0)$ \\
\hline $\mathrm{H}_{\text {Bridgehead }}$ & $4.4-4.5$ & $(0.0)$ & $(-0.1)-(0.1)$ & $(-0.2)-(0.2)$ & $(0.0)$ & $(0.0)-(0.1)$ \\
\hline
\end{tabular}

